\title{
Communications
}

\section{Performance Comparison on Three Time Delay Estimation Algorithms Using Experiments}

\author{
Junhao Li ${ }^{1}$, Wenhong Liu ${ }^{2}$ \\ ${ }^{1}$ School of Electronic and Information, Shanghai Dianji University, Shanghai, China \\ ${ }^{2}$ School of Electrical Engineering, Shanghai Dianji University, Shanghai, China
}

Email address:

1273889241@qq.com (Junhao Li), liuwenhong@sdju.edu.cn (Wenhong Liu)

To cite this article:

Junhao Li, Wenhong Liu. Performance Comparison on Three Time Delay Estimation Algorithms Using Experiments. Communications. Vol. 5, No. 3, 2017, pp. 24-28. doi: 10.11648/j.com.20170503.12

Received: June 28, 2017; Accepted: July 15, 2017; Published: August 7, 2017

\begin{abstract}
Time delay estimation (TDE) is applied in many areas. Its estimation performance plays an important role in many actual systems, such as malfunction sound location. In this paper, estimation performances of three TDE algorithms, correlation, covariance, and fractional lower order covariance, are compared. Traditional, additive noises in the actual collected signals are described by Gaussian distribution. However, they have often impulsiveness in practice, and are modeled as $\alpha$-stable distribution. First, correlation, covariance, and fractional lower order covariance method are analyzed in theory. Then, computer simulation experiments are carried out. Computer sound card records pure audio signals, different pulse intensity noises added to simulate actual environments. Next, results of three algorithms for time delay estimation were obtained in different signal to noise ratio (SNR) conditions. Under the same conditions, estimated RMS (root-mean-square) errors of three algorithms are analyzed and compared. Experimental results show that under low SNR and strong impulsive noise environments, fractional lower order covariance method indicates best estimation performance.
\end{abstract}

Keywords: Time Delay Estimation, $\alpha$-Stable Distribution, Impulsive Noise, Fractional Lower Order Covariance

\section{Introduction}

The time delay is the time difference between the homologous signals arriving at the receiver due to the difference in transmission distance and / or the difference of the transmission channel. Time delay estimation is usually based on parameter estimation and signal processing theory and method, the time delay estimated can determine other information, such as the channel temperature and structure, the signal source orientation, speed, distance $[1,11]$, direction of operation and other information [12]. Time delay estimation is a relatively active research topic in the field of signal processing. Its research puts forward new requirements for digital signal processing, adaptive filtering, time series analysis and correlation analysis to promote the development of disciplines. At the same time, the development of time delay estimation makes the system performance such as radar, sonar, biomedical engineering, communication and other engineering technology more stable, accurate and fast.

According to the characteristics of the signal to be processed, different statistical moments [2] can be used to analyze and process, and an algorithm suitable for the statistical moment of the signal can be designed. In the traditional time delay estimation study, the Gaussian model is widely used to study the time delay estimation algorithm because of its closed mathematical expression and mathematically easy to deduce, Such as correlation method, generalized correlation method [3], phase spectrum method, adaptive method and two spectrum and three-spectrum method [4, 5, 6, 7]. Through a deep study of the signal characteristics and theoretical analysis, the researchers put forward $\alpha$ stable distribution model which can better describe the signal characteristics. $\alpha$ stable distribution [16] is a generalized Gaussian model, which shows that there are infinitely many independent identities of infinite variance The distribution of the sum of the random variables must be $\alpha$ stable distribution, the smaller the value, the more significant the pulse of the sample. Gaussian distribution is a special case of it. Meanwhile, basing on the signal low-order statistical moment [14], there are have proposed covariant correlation method, fractional low-order covariance method, LMP 
adaptive method $[8,9,10]$.

In this paper, basing on the actual situation of the audio signal is often contained impulse noise, using $\alpha$ stable distribution to model additive noise, in different pulse intensity noise, theoretically analyzing three algorithms correlation method, covariant method, fractional low-order covariance method, then, computer simulation experiments. In this study, the computer sound card input pure audio signal, adding different pulse intensity noise, to simulate the actual situation. Under the condition of different SNR noise, the time delay estimation of the three algorithms is compared, and the root mean square error of the three algorithms is analyzed and compared under the same condition. The experimental results show that the fractional low - order covariance method has better performance in low SNR and strong impulse noise.

In the passive time delay estimation [13], single-path time delay estimation model is a two-element model (Figure 1). S is the signal source, the signal passes through two different channels to reach the two sensors in the space, and the received discrete signal model (Equation 1) can be expressed as

$$
\left\{\begin{array}{l}
X_{1}(n)=s(n)+v_{1}(n) \\
X_{2}(n)=g s(n-D)+v_{2}(n)
\end{array}\right.
$$

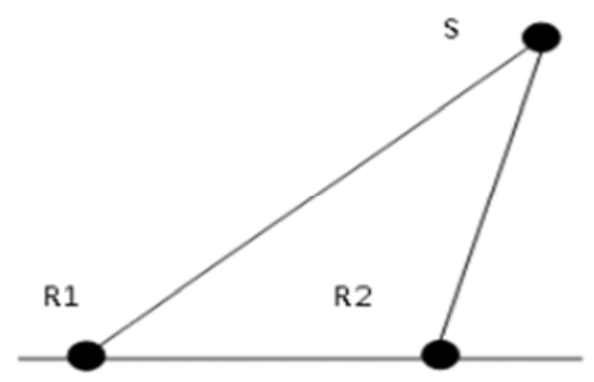

Figure 1. Two-senor model.

$\mathrm{S}$ (n) is the target source signal, D is the time delay true value, $g$ is the attenuation coefficient (general case $g=1$ ). And for receiving background noise

\subsection{Gaussian Model Correlation Algorithm}

Gaussian generalized density distribution function has closed expression, which is easy to be deduced mathematically. Most of the noise characteristics are also

\section{Basic Working Principle of Three Algorithms}

consistent with the Gaussian distribution process. Traditional time delay estimation method often modeled the noise as a Gaussian distribution, and the second order statistic is the main signal processing tool. Correlation analysis is a basic method of measuring the similarity of the two signals in the time domain. Presuming two received signals be described as the double primitive model described above, and the correlation function (formula 2 ) is

$$
R_{X 1 X 2}(\tau)=E\left[X_{1}(n) X_{2}(n+\tau)\right]
$$

the time delay is estimated. (formula 3)

$$
\hat{D}=-\arg \left\{\max \left[\hat{R}_{d}(m)\right]\right\}
$$

\subsection{Coherent Algorithm in Non - Gaussian Model}

In practical engineering applications [15], the collector's working environment is very complex, there is a lot of background noise, the space may contain the same as the thunderstorm pulse source, while the collector may also be a non-linear role. Due to the background noise and the reason of the acquisition device, the collected signal will often contain obvious impulsive noise. The statistical characteristics of the signal are deviated from the Gaussian model. The traditional correlation algorithm based on the Gaussian model is lacking in robust. So, according to the statistical characteristics of the noise signal to re-modeling, $\alpha$ stable distribution is a generalized Gaussian model, can better describe the noise in the pulse process.

The generalized central limit theorem is the generalization of the central limit theorem, which indicates that the distribution of the sum of infinite number of independent identically distributed random variables that may contain infinite variance must be stable. The smaller the value, the more impulsive the sample is. Expressed as follows:

If $X_{1}, X_{2}, \ldots, X_{n}$ is independent of the same distribution of random variables, when $\mathrm{n}$ tends to infinity

$$
X=\frac{X_{1}+X_{2}+\ldots+X_{n}}{a_{n}}-b_{n}
$$

$\mathrm{X}$ (Equation 3) is stable distribution. Where $a_{n} b_{n}$ are constant.

The covariance between the two signals is defined as (formula 5, 6)

$$
R_{C}(m)=E\left\{x_{2}(n)\left[x_{1}(n+m)\right]^{(p-1)}\right\}
$$

$$
R_{C}(m) \underline{\Delta}\left\{x_{2}(n)\left|x_{1}(n+m)\right|^{(p-1)} \operatorname{sgn}\left[x_{1}(n+m)\right]\right\} \quad 1 \leq p<\alpha
$$

In the actual calculation, because the statistical average of the signal is difficult to obtain, the commonly used method replaces the statistical average (formula 7,10 ) with a finite time average. The estimates of covariates can be obtained from n-point samples. 


$$
\hat{R}_{C}(m)=\frac{1}{N} \sum_{1}^{N} x_{2}(n)\left|x_{1}(n+m)\right|^{p-1} \operatorname{sgn}\left[x_{1}(n+m)\right] \quad 1 \leq p<2
$$

the time delay is estimated. (formula 8)

$$
\hat{D}=-\arg \left\{\max \left[\hat{R}_{C}(m)\right]\right\}
$$

The algorithm only applies to $1 \leq \alpha \leq 2$

\subsection{Fractional Low Order Covariance Algorithm}

The fractional low order covariance between the two signals is defined as (formula 9)

$$
\begin{gathered}
R_{C}(m)=E\left\{\left[x_{2}(n)\right]^{(A)}\left[x_{1}(n+m)\right]^{(B)}\right\} \\
\hat{R}_{d}(m)=\frac{1}{N} \sum_{1}^{N}\left|x_{2}(n)\right|^{A}\left|x_{1}(n+m)\right|^{B} \operatorname{sgn}\left[x_{1}(n+m) x_{2}(n)\right] \quad 0 \leq A<\frac{\alpha}{2} \quad 0 \leq B<\frac{\alpha}{2}
\end{gathered}
$$

the time delay is estimated. (formula 11)

$$
\hat{D}=-\arg \left\{\max \left[\hat{R}_{d}(m)\right]\right\}
$$

The algorithm only applies to $0<\alpha \leq 2$

It can be seen that the computational complexity of the correlation method is low and the algorithm is real-time. The covariant algorithm can select the appropriate value $p$ according to the pulse intensity in the noise. when $\alpha<1$ the method fails. The fractional low-order covariance algorithm is more operable for A and $\mathrm{B}$, and has better inhibitory effect on impulse noise and better practicability. From the calculation of the amount of comparison, the fractional low-order covariance method is larger. If $\alpha=2$, the covariance method and the fractional low-order covariance method become the correlation method, these two algorithms can be regarded as the generalization of the correlation method. Therefore, the generalized correlation method can also be transformed into a delay estimation algorithm suitable for impulsive noise environment.

\section{Signal Acquisition and Time Delay Estimation Simulation Experiment}

\subsection{Audio Signal Acquisition}

Using the computer sound card and microphone, enable the wavrecord function in matalab, set the sampling point to 1000 points, the sampling frequency is $8000 \mathrm{~Hz}$ and the data storage bit is 16 bit. Space environment no noise, the microphone obtain the spatial point sound source issued by a single channel audio signal. Another way the audio signal is obtained by shifting the acquisition signal, the number of shifts is 10 . The collected signal is processed as a pure signal, and the computer generates a stable sample ofastability, simulating the noise of different pulse intensity and signal to noise ratio. As a result, the "semi-real" sound source signal is obtained in accordance with the actual situation.

\subsection{Simulation Experiments}

In the impulsive noise environment ( $\alpha=2, \alpha=1.8$, $\alpha=1.5 \alpha=1.1$ ), the SNR is from $-6 \mathrm{~dB}$ to $3 \mathrm{~dB}$, adjust the parameters of the three delay estimation algorithms. The test data length is 1000 points, the experimental results are independent experiments 1000 times the statistical average. The root mean square error of the three algorithms is obtained under the same SNR. From the simulation results, Figure 2 shows the time-domain waveform of the signal to be processed when the signal-to-noise ratio is $3 \mathrm{~dB}(\alpha=2$, $\alpha=1.8, \alpha=1.5, \alpha=1.1$ ). It can be shown from the figure that the pulse performance of the processed signal is more pronounced when the $\alpha$ value is reduced. Figure 3 shows the root mean square error of the three algorithms for impulse noise at $-6 \mathrm{~dB}$ to $3 \mathrm{~dB}$ SNR. When the SNR is equal to $3 \mathrm{~dB}$, the estimated root mean square error is zero and the SNR is less than $3 \mathrm{~dB}$. The RMS of the fractional low order covariance method is the smallest, followed by the covariance method. Figure 4 is the impulsive noise ( $\alpha=1.8$ ), and the root mean square error estimated by the correlation method is much larger than the other two algorithms. The other two algorithms estimate the RMS error curve similarity, and the deviation of the lower order covariance method is close to zero. Figure 5 and Figure 6 are the impulse noise ( $\alpha=1.5, \alpha=1.1$ ), the correlation and covariance methods have a large root mean square error, but the fractional low order covariance still has a good estimated performance, the estimated root mean square error after the signal to noise ratio of $3 \mathrm{~dB}$ close to zero, And then the signal to noise ratio is less than $3 \mathrm{~dB}$, there is a better estimate of performance. Therefore, in the low signal-to-noise ratio and strong impulse noise environment, the fractional low-order covariance algorithm is more robust.

In the covariant algorithm and the fractional low-order covariance algorithm, the parameter selection is based on the existing literature. The choice of parameters of the two algorithms has great operability, and the optimal parameters will make the performance of the algorithm better. For the 
research algorithm and the actual engineering technology is of great significance.
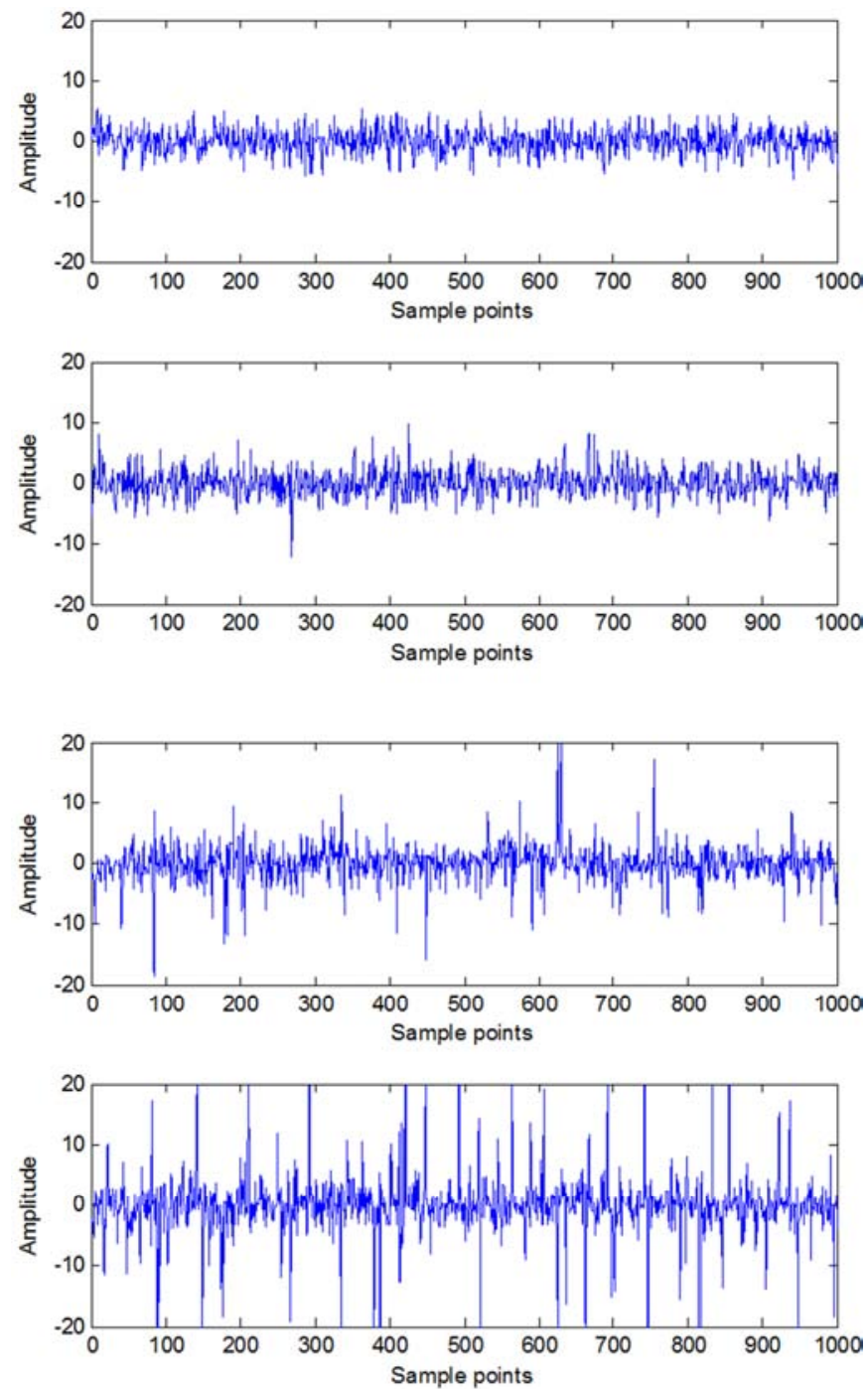

$\mathrm{A}=0.95 \mathrm{~B}=0.95 \mathrm{P}=1.9$

Figure 2. Signal time domain waveform added $\alpha=2, \alpha=1.8, \quad \alpha=1.5 \quad \alpha=1.1$ intensity pulse noise.

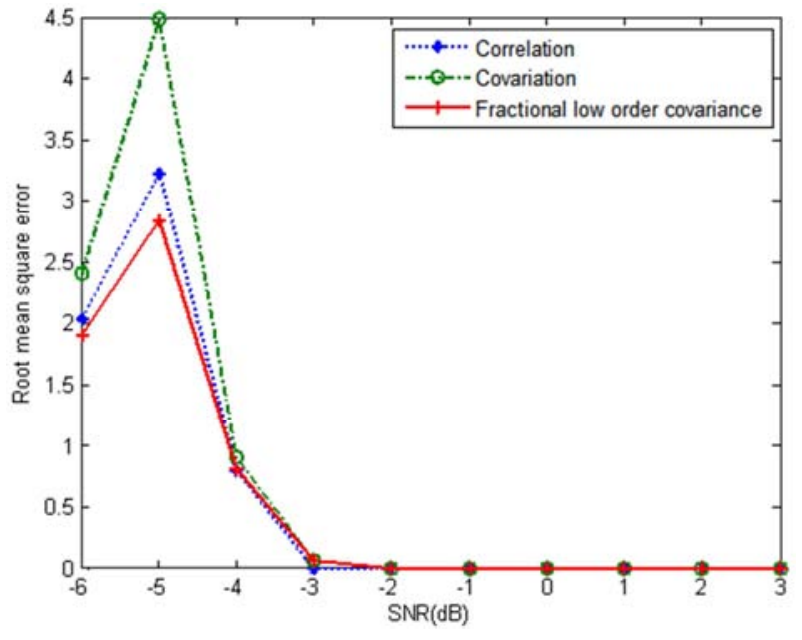

$\mathrm{A}=0.7 \mathrm{~B}=0.5 \mathrm{P}=1.5$

Figure 3. Correlation, covariance method, the fractional lower order covariance algorithms RMS estimated in different $\operatorname{SNR}(\alpha=2)$.

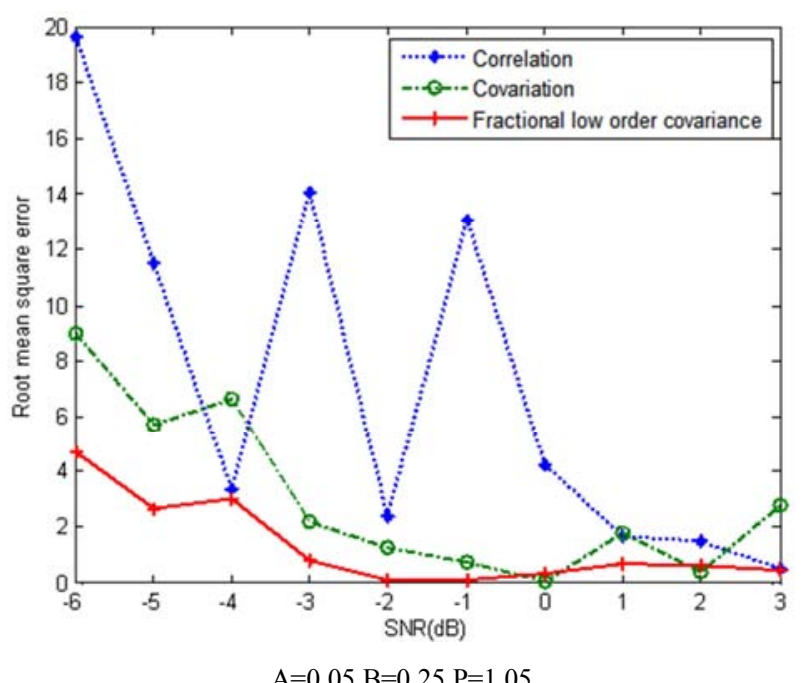

$\mathrm{A}=0.05 \mathrm{~B}=0.25 \mathrm{P}=1.05$

Figure 4. Correlation, covariance method, the fractional lower order covariance algorithms RMS estimated in different $\operatorname{SNR}(\alpha=1.8)$. 


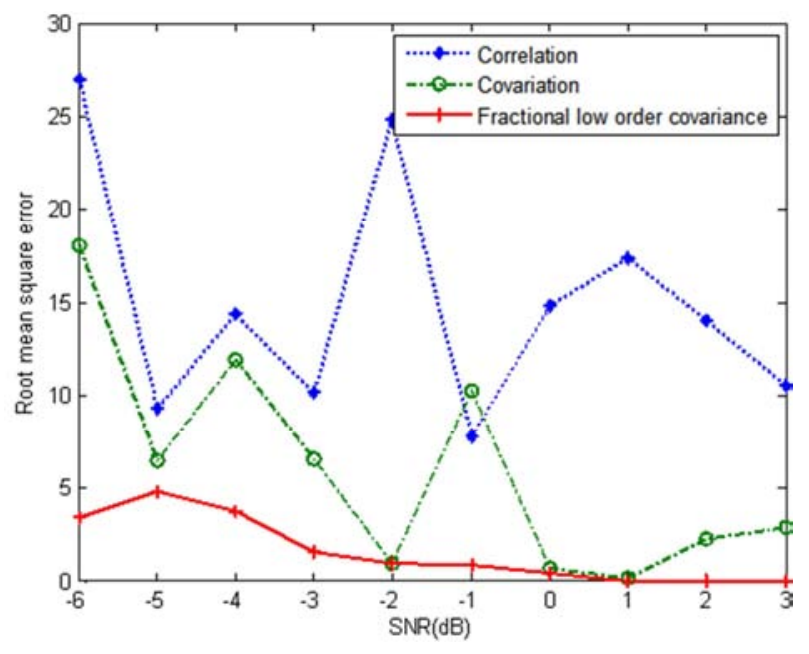

$\mathrm{A}=0.05 \mathrm{~B}=0.1 \mathrm{P}=0.05$

Figure 5. Correlation, covariance method, the fractional lower order covariance algorithms RMS estimated in different $\operatorname{SNR}(\alpha=1.5)$.

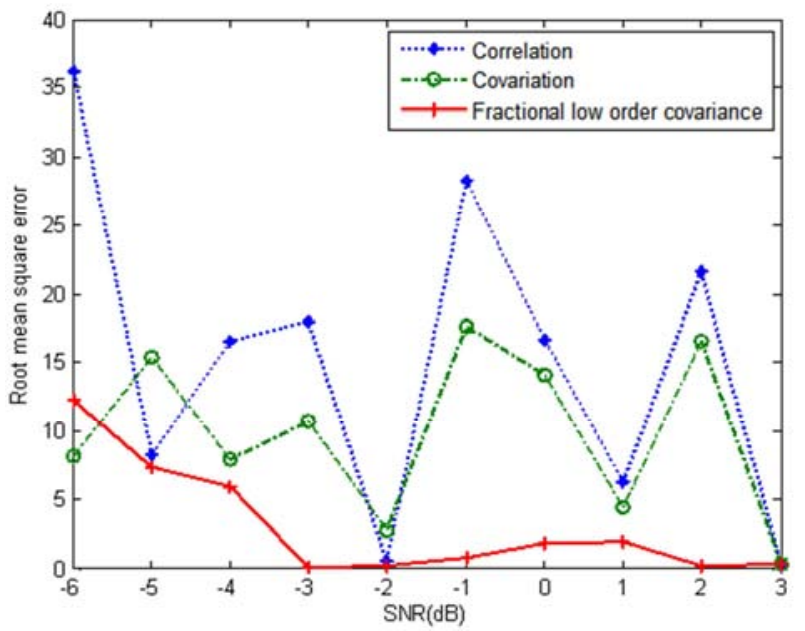

Figure 6. Correlation, covariance method, the fractional lower order covariance algorithms RMS estimated in different $\operatorname{SNR}(\alpha=1.1)$.

\section{Conclusion}

Time delay estimation is widely used in engineering fields such as radar, sonar, biomedical engineering, communication and so on. Its performance directly affects the working characteristics of the system. Analyzing the statistical characteristics of the noise are analyzed, and constructing the model of the applicable noisy. Then, designing and testing the algorithm in the real environment or simulate the problems. Based on TDE algorithm constructed by the $\alpha$ stabilization model, it is better robust to the Gaussian noise and non-Gaussian noise. Under the noise of the stronger pulse signal, the lower order covariance algorithm is better than the covariant algorithm. When $\mathrm{A}=\mathrm{B}=1$, the distribution is Gaussian distribution, the covariant and fractional low order covariance becomes the correlation algorithm. These two algorithms can be regarded as the generalization of the basic correlation method. Therefore, a variety of generalized correlation method can also be based on the situation to build a pulse signal noise for the delay estimation algorithm.

\section{References}

[1] Huo Hong. Passive positioning technology in the table tennis placement point estimation application [D]. Harbin Normal University, 2015.

[2] TANG Yong, Xiong Xingzhong. Comparison of Time Delay Estimation Algorithm Based on Fractional Lower Order Statistics [J]. Electronic Measurement Technology, 2014, (08): 65-69.

[3] LI Wei-hong, TANG Hai-bing, GONG Wei-guo. Study on Time Delay Estimation Method for Abnormal Sound Source Location in Public Places [J]. Journal of Instrumentation, 2012, (04): 750-756.

[4] Li Xuemei, Tao Ran, Wang Yue. Time delay estimation technology research [J]. Radar Science and Technology, 2010, (04): 362-371.

[5] ZHANG Duan-jin, ZHANG Zhong-hua, GUO Jian-jun, ZHANG De-jing. New method for time delay estimation based on fourth-order cumulant [J]. Journal of Zhengzhou University (Engineering Science), 2010, (01): 103-106.

[6] Yang Tao. Time Delay Estimation Method in Indoor Speech Source Location Technology [J]. Computer Engineering and Applications, 2009, (20): 246-248.

[7] Zhang Zhonghua, Zhang Duanjin, Guo Jianjun, Geng Yan. Multi-path delay estimation based on third-order cumulants [J]. Information and Electronic Engineering, 2009, (02): 94-98.

[8] LI Li-fang, LIU Qing-hua. Study on time delay estimation method under impulse noise [J]. Electro-acoustic Technology, 2008, (08): 57-59.

[9] Liu Wenhong. Time delay estimation method and application of impulse noise [D]. Dalian University of Technology, 2007.

[10] Wang Peng, Zhang Xiaotong, Xu Liyuan, He Jie, Xu Jinwu. High field near-field ranging algorithm based on adaptive time delay estimation [J]. Journal of Computers, 2016, (39): 1-19.

[11] Zhang Liang, Liu Zhiguang, Xiao Yanfan, Li Tiejun. Design of fault sound source location system for electromechanical equipment $[\mathrm{J}]$. Instrument Technique and Sensor, 2016, (02): 49-52.

[12] Li Ning, Cao Zhen, Deng Zhongliang, Han Ke. Time Estimation Method of Time Difference in Interference Source Location [J]. Systems Engineering and Electronics, 2016, (05): 994-997.

[13] Liu Xingyan. Research and application of generalized correlation delay estimation in passive time difference location (TDOA) [D]. Lanzhou Jiaotong University, 2015.

[14] Time delay estimation in unknown Gaussian spatially correlated noise. Nikias C L, Pan R. IEEE Trans. ASSP. 1988.

[15] Adaptive estimation of time delay in sampled system. Etter D M, Stearn S D. IEEE Transactions on Acoustics Speech and Signal Processing. 1981.

[16] Signal processing with Alpha-stable distributions. Nikias C L, Shao M. 1995. 